\title{
Meningoencefalitis Amebiana, revisión de tema.
}

\section{Amoebic Meningoencephalitis, review of literature.}

Pág. 132,143

Recibido: $14-05-2020$

Aceptado: 06-07-2020

Dra. Maribel Baraquiso Pazos ${ }^{1}$

Dra. Luisana Guier Bonilla ${ }^{2}$

Dr. Ariel Mendelewicz Montero ${ }^{3}$

1,2,3. Médico General, Trabajador Independiente. San José,Costa Rica.

\section{RESUMEN:}

Las infecciones del sistema nervioso central por amebas son una entidad poco frecuente, sin embargo, se caracterizan por una extrema virulencia y un pronóstico devastador. Naegleria fowleri es la ameba responsable de producir meningoencefalitis amebiana primaria. Los géneros Acanthamoeba y Balamuthia, otro tipo de amebas, son los agentes etiológicos de la encefalitis granulomatosa amebiana, que pese a tener un curso más lento y una menor virulencia presentan una letalidad casi tan elevada como la Naegleria fowleri. Debido a los síntomas inespecíficos, estas infecciones suelen ser simular cuadros bacterianos, virales $u$ otros no infecciosos. La alta sospecha diagnóstica sobre todo en pacientes con antecedente de exposición reciente con aguas contaminadas, resulta fundamental a la hora de abordar estos casos. Se han utilizado diferentes esquemas de tratamiento; no obstante, no se ha establecido una terapia eficaz y el desenlace sigue siendo fatal en la gran mayoría de los casos.

\section{PALABRAS CLAVE:}

Meningoencefalitis, Sistema Nervioso Central, Ameba, Infecciones Protozoarias del Sistema Nervioso Central.

\section{ABSTRACT:}

Amoebic infections of the central nervous system are a rare entity, yet they are characterized by extreme virulence and a devastating prognosis. Naegleria fowleri is the amoeba responsible for the development of primary amoebic meningoencephalitis. Acanthamoeba and Balamuthia genus, which are other types of amoeba, are the etiologic agents of amoebic granulomatous encephalitis. Despite having a slower course and being less virulent, these agents have a lethality almost as high 
as Naegleria fowleri. Due to the non-specific symptoms, these infections can simulate bacterial, viral or non infectious causes. High suspicion should be placed on this diagnosis, particularly when dealing with a history of recent exposure to possibly contaminated waters. Different treatment options have been used; however, effective therapy has not been established and the outcome remains fatal in the vast majority of cases.

\section{KEY WORDS:}

Meningoencephalitis, Central Nervous System, Ameba, Central Nervous System Protozoal Infections.

\section{Introducción:}

Las amebas de vida libre se encuentran alrededor de todo el globo terrestre (1). Los géneros Acanthamoeba, Balamuthia y Naegleria son las que pueden llegar a invadir el sistema nervioso central (SNC) y causar una enfermedad grave, y en la mayoría de los casos, con un desenlace letal $(2,3-6)$. A pesar de que su prevalencia a nivel mundial no es frecuente, su mortalidad es muy alta, con muy pocos sobrevivientes $(3,4$, 6-12).

Lasinfeccionesamebianasfuerondocumentadas en 1965 por Malcolm Fowler y Rodney Carter; sin embargo, no fue hasta 1966 que Calvin Butt, patólogo americano, utilizó por primera vez el término meningitis amebiana primaria después de describir tres infecciones asociadas a la exposición de aguas contaminadas (12).

Al ser una entidad sin datos diagnósticos característicos y poca frecuencia, los pacientes afectados dependen de una identificación expedita y pronto inicio de tratamiento. El rápido deterioro provocado por estas infecciones disminuye las posibilidades de recuperación, sólo un diagnóstico temprano podría llegar a evitar un desenlace fatal $(7,8)$.

Debido a la reciente aparición de nuevos casos de meningoencefalitis amebiana en Costa Rica, este tema cobra mayor relevancia. Por este motivo, el objetivo de la presente revisión bibliográfica se basa en caracterizar la meningoencefalitis amebiana y su cuadro clínico, con el fin de contribuir para que se establezca una sospecha clínica razonable y un diagnóstico oportuno, y de esta manera lograr la supervivencia de una mayor cantidad de pacientes.

\section{MÉTODOS:}

Para la redacción de esta revisión se realizó una búsqueda de artículos y publicaciones científicas en bases de datos como: The Journal of Infectious Diseases (JID), PubMed y Google Scholar con la frase "Meningitis amebiana, "Encefalitis por amebas" "Naegleria fowleri". Se tomaron en cuenta únicamente artículos en idiomas inglés y español, los cuales estuvieran indexados en revistas digitales con un Digital Object Identifier System (DOI). Todos los artículos utilizados fueron publicados en el período 2015-2020. En total se recopilaron 25 artículos, de los cuales únicamente se tomaron en cuenta 23. Los 2 artículos excluidos no contenían información clínicamente significativa para esta revisión.

\section{EPIDEMIOLOGÍA}

El organismo protista termofílico Naegleria fowleri (13), puede encontrarse en suelos y fuentes de aguas frescas como ríos, lagos y aguas termales $(4-6,8-10,12-15)$. Muy pocos casos se han hallado en aguas de piscinas cloradas y agua de grifo, (12). Se ha 
reportado su presencia en todas las partes del mundo, excepto en la Antártica $(9,14)$, con un predominio en los países tropicales $(3,4,15)$. Cabe recalcar que se han reportado casos en Missouri y Minnesota, por lo que su presencia en aguas templadas no es exclusiva $(11,12)$.

A la actualidad, se han reportado aproximadamente entre 300 y 440 casos de ME por Naegleria a nivel mundial desde su aparición en 1965. La mayoría de los casos han sido en Estados Unidos (EEUU), Australia y Francia $(4,6,10)$. En EEUU, se diagnostican aproximadamente 8 casos anuales $(11,16)$, con mayor frecuencia en la región sur de este país (12-14). Según reportes del Ministerio de Salud de Costa Rica, en la última década, se han registrado 3 casos de meningoencefalitis amebiana primaria, un caso en el 2014 y dos en el 2020, todos reportados en pacientes relacionados a la exposición a aguas termales $(17,18)$.

La incidencia ha aumentado globalmente, consecuencia del aumento de la temperatura global $(9,16)$. Es una infección que predomina durante el verano, sobre todo en la población joven masculina $(9,14)$, asociado con actividades acuáticas recreativas (14) y a la entrada del patógeno por vía nasal (8). Es una enfermedad subdiagnosticada debido a su corto período de incubación y su agresividad si no se trata de forma precoz $(5,6)$.

Por otra parte, los organismos del género Acanthamoeba son protozoarios más ubicuos encontrados en agua, suelo o aire, y se ha encontrado incluso en plantas nucleares, agua mineral embotellada, humidificadores, aire acondicionado y equipos de diálisis, entre otros $(3,4,7,9,12)$. Se han diagnosticado aproximadamente 150 casos a nivel global (4, 9 ). Además, puede ocurrir en cualquier época del año (9). Esta patología afecta principalmente, pero no de forma exclusiva, a pacientes inmunocomprometidos, con una prevalencia de hasta un $61 \%$ de los casos totales afectados por este microorganismo $(3,7,9)$.

Con respecto al agente de Balamuthia mandrillaris, se han reportado aproximadamente 200 casos alrededor del mundo, en todos los continentes excepto en África (4); en EEUU se han reportado en su mayoría en California, Texas y Arizona $(9,12)$. A diferencia de los patógenos mencionados anteriormente, este organismo no tiene predisposición en cuanto al estado inmunológico del huésped o clima de la región; además, tiene una prevalencia que se mantiene estable a lo largo de todo el año (9). Sin embargo, se ha visto que tiene una predominancia en pacientes hispanos, con hasta un $50 \%$ de los casos, probablemente por la combinación de factores como la susceptibilidad genética y la exposición ambiental $(4,12)$.

\section{ETIOLOGÍA:}

Se han descrito dos entidades clínicas distintas asociadas a la infección por las amebas; la meningoencefalitis amebiana primaria (MAP) causada por Naegleria fowleri, y la encefalitis granulomatosa amebiana (EGA), secundaria a infección por Acanthamoeba spp. o Balamuthia mandrillaris $(2,3,8,9,12,14,19,20)$. Dentro de las 40 especies del género Naegleria, $\mathrm{N}$. fowleri es la única que es patogénica en humanos y de los géneros de Balamuthia, la única especie capaz de producir una infección a nivel del SNC en las personas corresponde a B. mandrillaris $(3,6,12)$.

$\mathrm{N}$. fowleri es popularmente conocida como la "ameba come cerebros" $(5,8,12,15,21)$. Clásicamente se encontraba en áreas calientes;

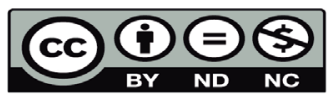


sin embargo, el cambio climático ha contribuido a su expansión geográfica $(3,4,8,12,14,15)$.

La MAP se caracteriza por tener una evolución aguda, fatal y fulminante, además suele presentarse en niños y adultos jóvenes previamente sanos, después de exponerse y tenercontactoconcuerpos deagua contaminados especialmente durante el verano $(2,4,5,8,12$, $14,15,21)$. Se desconoce el motivo por el cual existen personas expuestas diariamente a $\mathrm{N}$. fowleri que no presentan patología alguna y se continúan haciendo estudios para comprenderlo (12).

Por otro lado, la EGA se ha descrito tanto en pacientes inmunodeficientes como en pacientes inmunocompetentes; esto la convierte en una amenaza para la sociedad debido a su alta morbimortalidad $(2,7)$. Algunos de los pacientes más propensos a presentar una EGA son aquellos pacientes con patologías como $\mathrm{VIH}$, diabetes mellitus, lupus eritematoso sistémico. También en pacientes en tratamiento para cáncer o trasplantados de órganos que reciben terapia inmunosupresora (7).

\section{PATOGÉNESIS}

Existen dos formas patógenas el ciclo de vida de todas las amebas, los quistes y los trofozoítos. La forma quística se forma luego de exponerse a condiciones ambientales adversas $(3,7)$. Por otra parte, los trofozoítos son la forma activa del parásito, y son responsables de causar la infección invasiva en el ser humano $(9,22)$. Una particularidad de $\mathrm{N}$. fowleri, es que además de los trofozoítos y los quistes, también presenta una forma flagelada, que le permite sobrevivir en condiciones ambientales adversas $(3,6,8$, $9,15)$.
La MAP se produce por introducción accidental de $\mathrm{N}$. fowleri a través de la cavidad nasal después de sumergirse en agua contaminada con el parásito $(2,3-6,8,10,12,14,15$, 20-22). Tras un período de incubación de aproximadamente 6 días, inicia el proceso inflamatorio e infeccioso a nivel de $\operatorname{SNC}(4,5$, 14). Inicialmente, el microorganismo se adhiere a la mucosa respiratoria y a los filetes del nervio olfatorio.

Posteriormente, los trofozoítos invaden el SNC a través de la lámina cribosa del etmoides y producen una respuesta inflamatoria severa, con infiltración de macrófagos y neutrófilos $(2-6,8,10,12,14,15,20-22)$. Esto da como resultado un daño cerebral extenso asociado a lesiones líticas, hemorrágicas y necróticas, que pueden causar la muerte en menos de una semana $(2,4,8,20,21)$. Habitualmente, debido a su proximidad con el bulbo olfatorio, el lóbulo frontal es la región cerebral más afectada $(2,3$, $8,15,20)$.

Varios estudios han demostrado que la infección por $\mathrm{N}$. fowleri no se adquiere tras ingerir agua contaminada, ya que la única vía de transmisión descrita es la nasal $(12,15)$.

Otra de las particularidades de este microorganismo es la presencia de mecanismos y moléculas que le brindan capacidad para invadir el SNC y le permiten evadir la respuesta inmune. Algunos de ellos son los siguientes: la locomoción activa del microorganismo, su gran capacidad de adhesión a la mucosa nasal a través de proteínas de superficie, la producción de proteasas que destruyen el epitelio olfatorio y ciertos sustratos humanos como IgA, IgG, 
IgM, colágeno y fibronectina; además, causan la liberación de citoquinas proinflamatorias $(5,9$, 15, 22).

La MAP se caracteriza por tener una presentación aguda, a diferencia de la EGA, la cual es clásicamente una infección crónica, con un período de incubación de semanas a meses $(2,3,10)$.

La patogénesis de la EGA no está completamente comprendida; sin embargo, se cree que afecta el SNC por diseminación hematógena, distribuyéndose a través de la arteria cerebral media al lóbulo frontal, temporal y parietal $(3,9$, 20).

Los trofozoítos ingresan al torrente sanguíneo a través de lesiones en piel o del tracto respiratorio inferior y con menor frecuencia a través del los nervios olfatorios $(7,12,21)$. Una vez que el trofozoíto invade el SNC, se produce un granuloma necrotizante en el parénquima cerebral, con presencia de células gigantes multinucleadas, quistes, trofozoítos y una vasculitis fibrosa y necrotizante severa (4).

La Acanthamoeba posee la particularidad de que hospeda microorganismos endosimbiontes tales como: Naegleria chlamydophila, Legionella, Mycobacterium, Pseudomonas, Burkholderia candidatus, Paracaedibacter symbiosus, Betaproteobacterium, Amoebophilus asiaticus, Salmonella y Vibrio. Esta cualidad le permite a los microorganismos mencionados anteriormente, adaptarse a las células fagocíticas de los mamíferos y generar resistencias frente a condiciones ambientales adversas, aumentando así, la patogénesis de estos organismos (7).

A continuación se presenta una tabla con las principales diferencias entre la MAP y la EGA.

Tabla 1. Etiopatogenia de patógenos causantes de encefalitis amebiana. Tabla adaptada y modificada del artículo $(2-10,12,14,15,21)$.

\begin{tabular}{|c|c|c|}
\hline Patología & $\begin{array}{c}\text { Meningoencef } \\
\text { alitis amebiana } \\
\text { primaria }\end{array}$ & $\begin{array}{c}\text { Encefalitis } \\
\text { granulomatosa } \\
\text { amebiana }\end{array}$ \\
\hline $\begin{array}{c}\text { Agentes } \\
\text { etiológicos }\end{array}$ & $\begin{array}{c}\text { Naegleria } \\
\text { fowleri }\end{array}$ & $\begin{array}{c}\text { Acanthamoeba spp. } \\
\text { Balamuthia mandrillaris }\end{array}$ \\
\hline $\begin{array}{c}\text { Puerto de } \\
\text { entrada }\end{array}$ & $\begin{array}{c}\text { Cavidad nasal, } \\
\text { bulbo olfatorio }\end{array}$ & $\begin{array}{c}\text { Lesiones en piel, tracto } \\
\text { respiratorio inferior y } \\
\text { pocas veces bulbo } \\
\text { olfatorio }\end{array}$ \\
\hline $\begin{array}{c}\text { Tipo de } \\
\text { huésped }\end{array}$ & $\begin{array}{c}\text { Inmunocompete } \\
\text { nte }\end{array}$ & $\begin{array}{c}\text { Inmunocomprometido } \\
\text { Inmunocompetente }\end{array}$ \\
\hline $\begin{array}{c}\text { Factores } \\
\text { predisponentes }\end{array}$ & $\begin{array}{c}\text { Exposición a } \\
\text { aguas } \\
\text { calientes, } \\
\text { contaminadas }\end{array}$ & $\begin{array}{c}\text { Contacto con agua o tierra } \\
\text { contaminada, } \\
\text { inmunosupresión }\end{array}$ \\
\hline $\begin{array}{c}\text { Distribución } \\
\text { ambiental }\end{array}$ & $\begin{array}{c}\text { Aguas frescas } \\
\text { de ríos, lagos y } \\
\text { aguas termales }\end{array}$ & $\begin{array}{c}\text { Piscinas con agua } \\
\text { clorada, aire, tierra, aguas } \\
\text { termales, agua de ríos, } \\
\text { océanos y lagos }\end{array}$ \\
\hline $\begin{array}{c}\text { Período de } \\
\text { incubación }\end{array}$ & 5-7 dias & Semanas - meses \\
\hline
\end{tabular}

\section{MANIFESTACIONES CLÍNICAS:}

Las infecciones amebianas del SNC constituyen un cuadro de difícil diagnóstico. Estas meningoencefalitis (ME) se manifiestan de forma muy similar a infecciones virales, bacterianas o incluso tumores cerebrales $(4,9)$.

De forma general, las manifestaciones de la MAP y de la EGA son similares. Los síntomas iniciales suelen incluir cefalea, irritabilidad, náuseas, mareos y fiebre baja que oscila entre $37,5^{\circ} \mathrm{C}$ y $38,3^{\circ} \mathrm{C}$. Según la progresión y compromiso nervioso, otros síntomas pueden incluir alteración del estado mental, alucinaciones, confusión, déficits neurológicos focales, convulsiones, 
diplopía, parálisis de pares craneales, rigidez nucal o parálisis flácida de extremidades $(4,6$, $7,16,23)$. A continuación, se presentarán las manifestaciones clínicas específicas de cada entidad.

\section{MENINGOENCEFALITIS AMEBIANA PRIMARIA:}

Es un cuadro de infección aguda, rápidamente evolutiva que puede causar la muerte en menos de 5 días $(3,9)$. Esta dramática evolución parece relacionarse con la invasión de la cavidad nasal y rápido acceso a las estructuras nerviosas centrales a través del bulbo olfatorio $(3,8,15$, 21). Lo anterior conlleva a edema, necrosis, ME hemorrágica fulminante, leptomeningitis fibrinopurulenta y herniación de estructuras cerebrales, que en muchas ocasiones simula una $M E$ bacteriana fulminante $(4,9,14,21)$.

Una casuística del 2020 compuesta de 256 pacientes con diagnóstico de MAP, clasificó sus síntomas de acuerdo su presentación ya sea temprana o tardía. Los síntomas predominantes en la etapa temprana fueron los pródromos catarrales; mientras que la presentación tardía se caracterizó por presentar síntomas de compromiso neurológico $(8,14)$.

Tabla 2. Síntomas de presentación más comunes en pacientes con MAP $(4,14)$

\begin{tabular}{|c|c|}
\hline Síntoma reportado & $\begin{array}{c}\text { \% de } \\
\text { aparición }\end{array}$ \\
\hline Fiebre $\left(38,5-41^{\circ} \mathrm{C}\right)$ & 88 \\
\hline $\begin{array}{c}\text { Cefalea bifrontal o } \\
\text { bitemporal }\end{array}$ & 82 \\
\hline $\begin{array}{c}\text { Náuseas y vómitos } \\
\text { Alteración del estado } \\
\text { mental }\end{array}$ & 57 \\
\hline Rigidez nucal & 35 \\
\hline Coma & 13 \\
\hline
\end{tabular}

Otros hallazgos observables son: fotofobia, letargia, estupor, convulsiones y anomalías del comportamiento, principalmente irritabilidad e inquietud. La diplopía y parálisis de pares craneales III, IV y VI se relacionan con edema cerebral y herniación inminente (4).

Por su parte, el cuadro en niños suele iniciar con rinorrea hialina, disgeusia y alteraciones del olfato luego de 2-5 días de la exposición a fuentes de agua. Posteriormente evoluciona a fiebre, cefalea intensa, vómitos, irritabilidad y un resultado fatal en el transcurso de 7-10 días (21).

Meningoencefalitis Granulomatosa amebiana:

Esta entidad tiene una evolución progresiva, llegando a ser mortal tras 2-3 meses tras su inicio, esto debido a la hipertensión intracraneana (HIC) $(4,7,9)$. Se presenta como una encefalitis subaguda-crónica, que cursa con cefalea, meningismo, alteración del estado mental y del comportamiento, afectación del sensorio y déficits neurológicos focales, tales como parálisis de pares craneales, hemiparesias y afasias). Además se acompaña de otros síntomas como fiebre, náuseas, fotofobia, pérdida de la visión y una aparición temprana de convulsiones $(4,12$, 21).

Los casos de Balamuthia pueden tener 2 presentaciones, algunos pacientes desarrollan una lesión cutánea inicial descrita como lesiones ulcerativas crónicas o nódulos eritematosos que preceden el deterioro neurológico semanas a meses después; mientras que otra parte hace el debút con síntomas neurológicos hasta 30 días después de la exposición $(4,9)$.

Estos agentes poseen la capacidad de simular síndromes vasculares como aneurismas $u$

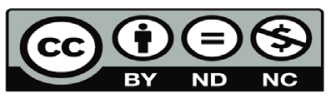


oclusiones vasculares cerebrales debido al daño endotelial y angeítis necrotizante que producen. Esto lleva a una mayor inflamación en el parénquima cerebral, formación de granulomas y aumento de permeabilidad vascular y finalmente, necrosis tisular $(3,9,21)$.

\section{DIAGNÓSTICO}

La MAP debe considerarse en aquellos pacientes que presenten una ME clínica y que tengan el antecedente de exposición a aguas contaminadas con el microorganismo; además de la presencia de un líquido cefalorraquídeo (LCR) purulento, pero con ausencia de bacterias (21). El diagnóstico temprano es sumamente importante, aunque puede ser retrasado por la ausencia de signos específicos (4); esto lleva a que tan solo un $27 \%$ de los casos se diagnostiquen antemortem $(5,8,9)$, por lo tanto, la sospecha diagnóstica se torna verdaderamente relevante.

Como cualquier otra entidad clínica, el diagnóstico de una MAP amerita una anamnesis y un examen físico exhaustivo. La historia clínica constituye un pilar fundamental, que debe indagar acerca del contacto reciente con fuentes de agua contaminadas (5). Asimismo, para orientar el diagnóstico es necesario realizar un análisis de LCR (4-6, 8-10, 21, 22), en el cual se observarán características tales como:

- Leucocitosis con predominio de polimorfonucleares al inicio de la enfermedad.

- Hipoglucorraquia.

- Presión de apertura aumentada, que puede llegar a los $600 \mathrm{mmH} 2 \mathrm{O}$.

- Presencia aumentada de glóbulos rojos.

- Presencia de trofozoítos en el LCR, con tinciones de Wright-Giemsa.

- Coloración grisácea-amarillento al inicio de la enfermedad, y en fases más avanzadas tinción de color rojo por el aumento de eritrocitos.

Se ha considerado una tríada diagnóstica, la cual se compone de: aumento de leucocitos en LCR, negatividad del cultivo Gram e historia de exposición a aguas frescas (9). A pesar de lo mencionado anteriormente, la reacción en cadena de polimerasa (PCR) se considera el método de elección para el diagnóstico del MAP, ya sea en LCR o en tejido cerebral $(8,16)$.

Las pruebas de imagen se consideran inespecíficas (4); sin embargo, existen hallazgos que pueden orientar el diagnóstico de una MAP. Sin importar la técnica de imagen que se utilice, se ha visto una mayor prevalencia de lesiones en el lóbulo frontal (3). Ahora bien, en la tomografía axial computarizada (TAC) y en la resonancia magnética (RM) se pueden encontrar obliteraciones de las cisternas en el mesencéfalo y el espacio subaracnoideo, y lesiones con reforzamiento periférico (Figura 1) $(9,21,22)$. Además, se pueden observar alteraciones a nivel del SNC, borramiento de los surcos corticales, herniación del encéfalo, exudados meníngeos, infartos hemorrágicos, edema cerebral y realce leptomeníngeo, entre otros $(5,6,21)$. Se ha descrito en la literatura la presencia de una infección focal única, con edema cerebral difuso y signos de HIC tales como desviación de la línea media y borramiento de los ventrículos y cisternas cerebrales (3).

Por otro lado, la EGA se diagnostica de forma muy similar, pero tiene ciertas características distintivas que se expondrán a continuación. Los hallazgos de las técnicas de imagen son inespecíficos y se ha encontrado mayor afectación, al igual que en las MAP, en el lóbulo frontal $(3,9)$. Este tipo de meningitis presenta dos patrones que se pueden hallar tanto en la

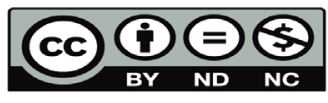


TAC o como en la RM: uno de ellos es un patrón multifocal con presencia de múltiples lesiones con realce en forma de anillo y edema perilesional; $y$ el otro constituye un patrón pseudotumoral que asemeja una masa grande y solitaria $(3,9,21)$.

En cuanto al análisis de LCR, se observa pleocitosis y leucocitosis, con una glucosa que puede estar dentro de los rangos normales o disminuida, y una elevación de moderada a severa de las proteínas. Es raro encontrar los trofozoítos, por lo que el diagnóstico se hace mediante tejido de las lesiones en piel o por material obtenido en biopsias cerebrales $(9$, 21). Sin embargo, al igual que en la MAP, el diagnóstico definitivo se realiza por medio de $\mathrm{PCR}$, con el aislamiento de especies del género Acanthamoeba y B. mandrillaris $(4,9)$.

Es importante tener en cuenta diagnósticos diferenciales como por ejemplo: neoplasias cerebrales, esclerosis múltiple, encefalitis por lupus eritematoso sistémico, leucoencefalopatía multifocal progresiva, infartos, meningitis por otras causas (viral, tuberculosa, bacteriana) o toxoplasmosis cerebral (3).

\section{TRATAMIENTO:}

A pesar de que ninguna terapia ha mostrado ser efectiva en el manejo de estas infecciones, y que los tratamientos disponibles presentan una respuesta desfavorecedora, el objetivo es administrar de forma temprana el fármaco para aumentar su eficacia $(3,15,21)$.

Dificultades en procesos de farmacocinética y farmacodinamia hacen que muchos de los agentes actualmente disponibles no logren cruzar la barrera hematoencefálica (BHE) en concentraciones efectivas para combatir la infección. Algunos de ellos requieren de dosis altas para ser efectivos y la neurotoxicidad producida impide su uso, por esta razón es que algunos autores han intentado el uso de estos fármacos por vía intratecal. Otro obstáculo para la terapia es que en lugar de ser fármacos bactericidas, son bacteriostáticos $(3,7)$. Los reportes en la literatura apoyan el uso de terapia combinada con el objetivo de aumentar la eficacia al utilizar mecanismos de acción diferentes (7, 12). La combinación más efectiva y esquema de tratamiento depende más de la tolerancia del paciente, edad, género y condiciones médicas que de su efectividad demostrada $(3,15)$.

La Sociedad de Enfermedades Infecciosas de América (IDSA) recomienda el uso combinado de TMP-SMX, Rifampicina y Ketoconazol o Fluconazol junto a Sulfadiazina y Pirimetamina para los casos de Acanthamoeba $(7,12)$. Sin embargo, Anfotericina B, macrólidos, Miltefosine, Flucitosina, Sulfadiacina y Pentamidina también han sido utilizados (4). La combinación más exitosa parece incluir: Anfotericina B, TMP-SMX y Rifampicina (3).

El caso de EGA por Balamuthia, se han reportado estudios de 4 sobrevivientes los cuales fueron tratados con Pentamidina, Isetionato, Fluconazole, Flucitosina, Sulfadiazina y un macrólido $(3,9,12)$.

El tratamiento de MAP se enfoca en la eliminacion de la ameba y en la neuroprotección, principalmente en manejo de la HIC (ventriculostomías, terapia hiperosmolar o hiperventilación), hipotermia terapeútica y uso de esteroides $(8,9)$. El objetivo anti-ameba combate con Anfotericina $\mathrm{B}$ el cual es el medicamento de elección $(4,8)$ asociado a compuestos azolados, Sulfadiazina y Rifampicina $(3,21)$. En una revisión que estudió a 7 sobrevivientes con 
PAM, se demostró que todos fueron tratados con Anfotericina B intravenosa (IV) y 5 con Anfotericina B intratecal. Asimismo, 6 de ellos fueron tratados con compuestos azolados y Rifampicina; 5 de ellos con Dexametasona y 4 recibieron Azitromicina y Miltefosina $(4,14)$.

\section{PRONÓSTICO:}

De forma general, el pronóstico tanto de MAP y EGA es reservado. Ambas entidades terminan siendo fatales en la gran mayoría de casos, aún con tratamiento. Una distinción de lo anterior es que los casos de EGA suelen ser mortales en 1-2 meses $(3,7)$. En la MAP el desenlace se da en 7-10 días (4).

La gran mayoría de pacientes infectados por Acanthamoeba fallece (97-98\%) (3,7). El caso de Balamuthia no es la excepción con mortalidades que rondan el $98 \%$ (12). Infecciones de Naegleria tiene reportes de $95-97 \%$ de mortalidad (8-11).

Una publicación en 2017 reportó sobrevida del $5 \%$ para estas infecciones (3). La cifra se relaciona de forma directa con diagnóstico e intervención temprana, tratamiento agresivo y manejo de la HIC (8).

\section{Conclusiones:}

Las infecciones amebianas con tropismo por el SNC son una patología que afecta gravemente al ser humano, y en la mayoría de los casos se consideran entidades letales.

La principal manifestación a nivel de SNC puede ser clasificarse según su etiología, la MAP causada por $\mathrm{N}$. fowleri, y la EGA que puede ser causada por organismos del género Acanthamoeba y la especie Balamuthia mandrillaris.

Es importante recalcar que el entendimiento de los mecanismos patogénicos de la infección amebiana podría explicar cuáles factores del huésped hacen que sea susceptible al contagio, teniendo en cuenta que son organismos ubicuos que rara vez causan patología en el ser humano.

Tanto la MAP como la EGA constituyen enfermedades con evoluciones muy tórpidas, y debido a su poca prevalencia e inespecificidad muy pocas veces se toman en cuenta en los diagnósticos diferenciales. Por lo tanto, ya que el diagnóstico precoz puede influir en la sobrevida del paciente, es importante tomar en cuenta en todo paciente con meningitis y la tríada diagnóstica de aumento de leucocitos en LCR, negatividad del cultivo Gram y antecedente de exposición a aguas frescas potencialmente contaminadas, la posibilidad de que presente una ME amebiana.

A pesar de la búsqueda de un medicamento que sea eficaz para combatir los casos de ME amebiana, actualmente no existe una terapia estandarizada y la mortalidad es muy alta, con casos excepcionales de sobrevivientes.

Esto puede deberse a la poca prevalencia y subdiagnóstico de la enfermedad, ya que la mayoría de los casos se diagnostican postmortem. Por lo que estudios futuros a fin de definir esto son necesarios.

\section{Conflictos de Interés}

Los autores declaran ningún conflicto de interés. 


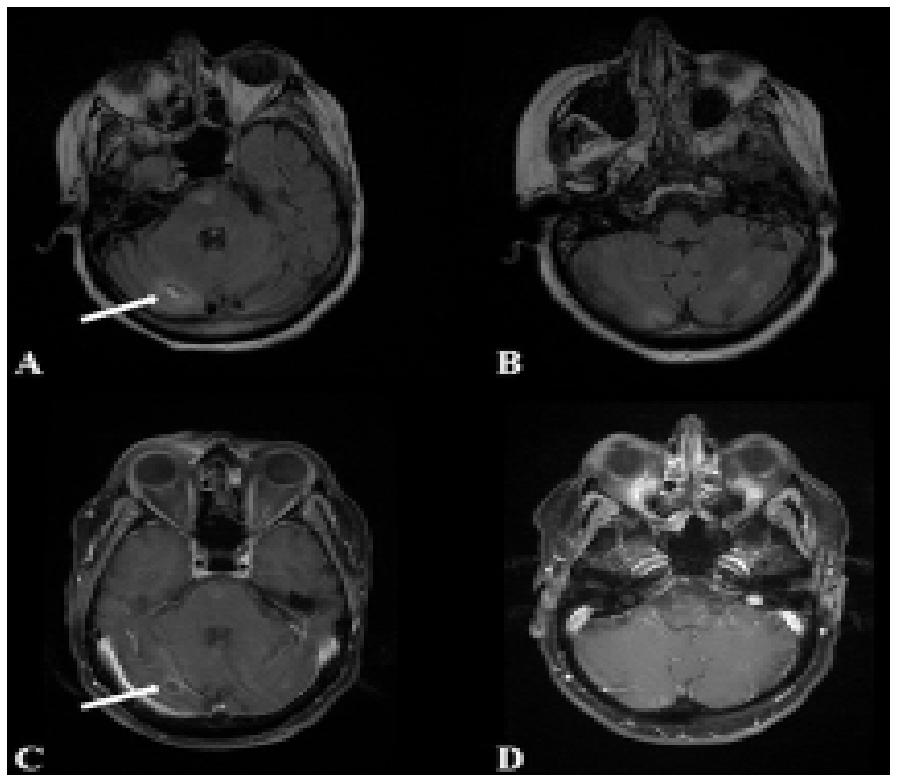

Figura 1. RM de cerebro que muestra lesiones con reforzamiento periférico ( $\mathrm{A}$ y $\mathrm{C}$ ), provocadas por Balamuthia mandrillaris. Imagen tomada del artículo "The evolving spectrum of amoebic meningoencephalitis" (9).

\section{BLIBLIOGRAFÍA}

1. Kenfak A, Eperon G, Schibler M, Lamoth F, Vargas MI, Stahl JP. Diagnostic approach to encephalitis and meningoencephalitis in adult returning travelers. Clin Microbiol Infec. 2019; 25 (4): 415-421. https://doi.org/10.1016/j.cmi.2019.01.008

2. Garcia HH, Nath A, Del Brutto OH. Parasitic Infections of the Nervous System. Semin Neurol. 2019; 39 (3): 358-368. https://doi.org/10.1055/s-0039-1693036.

3. Ong T, Khan N, Siddiqui R. Brain-Eating Amoebae: Predilection Sites in the Brain and Disease Outcome. J Clin Microbiol. 2017; 55 (7): 1989-1997. https://doi.org/10.1128/JCM.02300-16

Król-Turmińska K, Olender A. Human infections caused by free-living amoebae. Ann Agric En-

4. viron Med. 2017; 24 (2): 254-260. https://doi.org/10.5604/12321966.1233568

Martínez-Castillo M, Cárdenas- Zúñiga R, Coronado-Velázquez D, Debnath A, Serrano-Luna 5. J, Shibayama M. Naegleria fowleri after 50 years: is it a neglected pathogen?. J Med Microbiol. 2016; 65 (9): 885-896. https://doi.org/10.1099/jmm.0.000303

Bellini NK, Santos TM, da Silva MTA, Thiemann $\mathrm{OH}$. The therapeutic strategies against Naegle-

6. ria fowleri. Exp Parasitol. 2018; 187:1-11. https://doi.org/10.1016/j.exppara.2018.02.010

Kalra S, Sharma P, Shyam K, Tejan N, Ghosal U. Acanthamoeba and its pathogenic role in gra7. nulomatous amebic encephalitis. Exp Parasitol. 2020; 208: 107788. https://doi.org/10.1016/j. 
exppara.2019.107788

8. Cooper AM, Aouthmany S, Shah K, Rega PP. Killer amoebas: Primary amoebic meningoencephalitisin a changing climate. JAAPA. 2019; 32 (6): 30-35. https://doi.org/10.1097/01. JAA.0000558238.99250.4a

9. Gompf SG, Garcia C. Lethal encounters: The evolving spectrum of amoebic meningoencephalitis. IDCases. 2019; 15:e00524. https://doi.org/10.1016/j.idcr.2019.e00524

10. Dzikowiec M, Góralska K, Blaszkowska J. Neuroinvasions caused by parasites. Ann Parasitol. 2017; 63 (4): 243-253.https://doi.org/10.17420/ap6304.111

11. Cope J, Murphy J, Kahler A, et al. Primary Amebic Meningoencephalitis Associated With Rafting on an Artificial Whitewater River: Case Report and Environmental Investigation. Clin Infect Dis. 2018; 66 (4): 548-553. https://doi.org/10.1093/cid/cix810

12. Shih R, Koeller K. Bacterial, Fungal, and Parasitic Infections of the Central Nervous System: Radiologic-Pathologic Cor- relation and Historical Perspectives. Radiographics. 2015; 35 (4): 1141-1169. https://doi.org/10.1148/rg.2015140317

13. Zhang LL, Wu M, Hu BC, et al. Identification and molecular typing of Naegleria fowleri from a patient with primary amebic meningoencephalitis in China. Int J Infect Dis. 2018; 72: 28-33. https://doi.org/10.1016/j.jijid.2018.05.001

14. Gharpure R, Bliton J, Goodman Ali I, Yoder J, Cope J. Epidemiology and Clinical Characteristics of Primary Amebic Meningoencephalitis Caused by Naegleria fowleri: A Global Review. Clin Infect Dis. 2020; pii:ciaa520. https://doi.org/10.1093/cid/ciaa520

15. Jahangeer M, Mahmood Z, Munir N, et al. Fowleri: sources of infection, pathophysiology, diagnosis, and management; a review. Clin Exp Pharmacol Physiol. 2020; 47 (2): 199-212. https:// doi.org/10.1111/1440-1681.13192

16. Cope JR, Ali IK. Primary Amebic Meningoencephalitis: What Have We Learned in the Last Five Years?. Curr Infect Dis Resp. 2016; 18 (10): 31. https://doi.org/10.1007/s11908-016-0539-4

17. Ministerio de Salud. "Exigen rotulación de advertencia, altura máxima y prohibición de toboganes que desboquen en aguas termales" [Internet]. San José, Costa Rica: Ministerio de Salud; 2020 [revisado el 25 de junio del 2020]. Disponible en https://www.ministeriodesalud. go.cr/index.php/centro-de-prensa/noticias/741-noticias-2020/1541-exigen-rotulacion-de-advertencia-altura-maxima-y-prohibicion-de-toboganes-que-desboquen-en-aguas-termales.

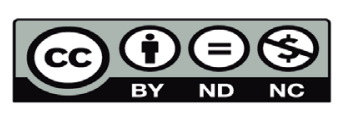


18. Ministerio de Salud. "Confirmado extraño caso de Meningitis amebiana primaria en joven de 15 años" [Internet]. San José, Costa Rica: Ministerio de Salud; 2020 [revisado el 25 de junio del 2020]. Disponible en https://www.ministeriodesalud.go.cr/index.php/centro-de-prensa/ noticias/741-noticias-2020/1528-confirmado-extrano-caso-de-meningitis-amebiana-primaria-en-joven-de-15-anos

19. Rapalino O, Mullins ME. Intracranial Infectious and Inflammatory Diseases Presenting as Neurosurgical Pathologies. Neurosurgery. 2017; 81 (1): 10-28. https://doi.org/10.1093/neuros/ nyx201

20. Baig AM. Pathogenesis of amoebic encephalitis: Are the amoebae being credited to an 'inside job' done by the host immune response?. Acta Trop. 2015;148:72-76. https://doi.org/10.1016/j. actatropica.2015.04.022

21. Singhi P, Saini AG. Fungal and Parasitic CNS Infections. Indian J Pediatr. 2019; 86 (1): 83-90. https://doi.org/10.1007/s12098-017-2487-x

22. Grace E, Asbill S, Virga K. Naegleria fowleri: Pathogenesis, Diagnosis, and Treatment Options. 2015; 59 (11): 6677-6681. https://doi.org/10.1128/AAC.01293-15

23. Chen M, Ruan W, Zhang L, Hu B, Yang X. Primary Amebic Meningoencephalitis: A Case Report. Korean J Parasitol. 2019; 57 (3): 291-294. https://doi.org/10.3347/kjp.2019.57.3.291 